\title{
ON THE CHARACTERISTIC FUNCTION OF A MEROMORPHIC FUNCTION I
}

\author{
HARI SHANKar \\ (Received September 20, 1956 ; revised April 15, 1957)
}

1. Introduction. Let $f(z)$ be meromorphic for $|z|<\infty$ and

$$
T(r)=\int_{0}^{r} S(t) d t
$$

where

$$
S(r)=-\frac{1}{\pi} \int_{0}^{r} \int_{0}^{2 \pi}\left(\begin{array}{c}
\left|f\left(t e^{i \theta}\right)\right| \\
1+\left|f\left(t e^{i \theta}\right)\right|^{2}
\end{array}\right)^{2} t d t d \theta
$$

be its Nevanlinna characteristic function in "Spherical Normal" form $[2 ; \mathrm{p}$. 177] and

$$
\limsup _{r \rightarrow \infty} \frac{\log T(r)}{\log r}=\rho(0 \leqq \rho \leqq \infty)
$$

be its order. If $0<\rho<\infty$

$$
\begin{aligned}
& \alpha \\
& \beta
\end{aligned}=\lim _{r \rightarrow \infty}\left\{\begin{array}{l}
\sup \\
\inf
\end{array}\right\} \frac{T(r)}{r^{p}} ; \quad \gamma=\lim _{r \rightarrow \infty}\left\{\begin{array}{l}
\sup \\
\text { inf }
\end{array}\right\} \frac{S(r)}{r} .
$$

S. K. Singh $[3 ;$ p. 10 , Pt 2 . $]$ has established the following results

$$
\begin{aligned}
& \text { (i) } \delta \leqq \rho \beta \leqq \delta\left(1+\log \frac{\gamma}{\delta}\right) \\
& \text { (ii) } \delta \leqq \frac{\gamma}{e} e^{\delta} \bar{\gamma} \leqq \rho \alpha \leqq \gamma \\
& \text { (iii) } \gamma+\delta \leqq e \rho \alpha .
\end{aligned}
$$

The object of this note is to establish results which include the above as special cases. I compare the grow th function with a more general function $r^{p} L(r)$ where $L(x)$ is a "slowly changing" function; i. e. $L(x)>0$ and continuous for $x \geqq x_{0}$ and $L(c x) \sim L(x)$ as $x \rightarrow \infty$, for every constant $c>0$. ( $L(x)$ need not tend to infinity.) The following results for such functions are worth mentioning [1] which are employed here too.
(a) For any $\lambda>0, x \rightarrow \infty$$$
x^{-\lambda} L(x) \rightarrow 0 ; x^{\lambda} L(x) \rightarrow \infty
$$
(b) $\int_{\mathbf{i}}^{u} x^{\lambda-1} L(x) d x \sim L(u) \frac{u^{\lambda}}{\lambda}$ 
(c) $\int_{u}^{\infty} x^{-\lambda-1} L(x) d x \sim L(u) \frac{u^{-\lambda}}{\lambda}$

2. We set

$$
\tau^{\tau}=\lim _{r \rightarrow \infty}\left\{\begin{array}{l}
\sup \\
\inf
\end{array}\right\} \frac{T(r)}{r^{r} \mathcal{L}(r)} ; \quad \mu=\lim _{r \rightarrow \infty}\left\{\begin{array}{l}
\sup \\
\text { inf }
\end{array}\right\} \frac{S(r)}{r^{r} L(r)}
$$

and obtain the following results.

THEOREM. If $0<\rho<\infty$

$$
\begin{aligned}
& \text { (i) } \quad \nu \leqq \rho t \leqq \nu\left(1+\log \frac{\mu}{\nu}\right) \leqq \mu ; \nu \neq 0 \\
& \text { (ii) } \nu \leqq \frac{\mu}{e} e^{\nu / \mu} \leqq \rho T \leqq \mu \leqq e \rho \tau
\end{aligned}
$$

and in particular $\mu+\nu \leqq e \rho \tau$. Obviously if there is equality in $\mu \leqq e \rho \tau ; \nu=0$.

CoROLlary. $\mu=\nu$ if and only if $\nu=\rho T$. Consequently equality can not hold simultaneously in $\nu \leqq \rho \tau$ and $\mu+\nu \leqq e \rho \tau$ and hence a fortiori it can never hold simultaneously in $\mu \leqq e \rho \tau$ and $\nu \leqq \rho \tau$ if $\tau>0$. then

Proof. (i) Let $R=r k^{1 / \rho}$ where $k \geqq 1$ is an arbitrary number. If $\nu<\infty$

$$
\begin{aligned}
T(R) & =K_{1}+\left(\int_{r_{0}}^{r}+\int_{r}^{R}\right) \frac{S(t)}{t} d t \\
& >K_{1}+(\nu-\varepsilon) \int_{r_{0}}^{r} t^{\rho-1} L(t) d t+S(r) \int_{r}^{R} \frac{d t}{t} \\
& \sim(\nu-\varepsilon) \frac{L(\boldsymbol{r})}{\rho} r^{\rho}+\frac{S(\boldsymbol{r})}{\rho} \log k .
\end{aligned}
$$

Therefore

$$
k \frac{T(R)}{R^{\prime} L(R)}>\frac{(\nu-\varepsilon)}{\rho}+\frac{S(r)}{r^{\rho} L(r)} \cdot \frac{\log k}{\rho}
$$

Hence we get

$$
\rho_{k} \tau \geqq \nu+\mu \log k
$$

and

$$
\rho_{k} t \geqq \nu(1+\log k) .
$$

On the other hand (2.1) gives for $\mu<\infty$

$$
T(R)<K_{1}+(\mu+\varepsilon) \int_{r_{0}}^{r} t^{\rho-1} L(t) d t+S(R) \int_{r}^{R} \frac{d t}{t} .
$$

Whence we get

$$
\rho_{k} \tau \leqq \mu(1+k \log k)
$$


and

$$
\rho_{k} t \leqq \mu+\nu_{k i} \log k
$$

Which also hold when $\mu=\infty$.

Divide (2.5) by $k$, then the right hand side of the new inequality has a minimum when $k=\mu / \nu,(\nu \neq 0)$ and we get

$$
\rho t \leqq \nu\left(1+\log \frac{\mu}{\nu}\right) \text {. }
$$

Taking $k=1$ in (2.3) we get $\nu \leqq \rho t$.

Further, since $1+\log x \leqq x$ for all $x$ we obtain finally

$$
\nu \leqq \rho t \leqq \nu\left(1+\log \frac{\mu}{\nu}\right) \leqq \nu \frac{\mu}{\nu}=\mu .
$$

Proof (ii). Take $k=\exp \left(1-\frac{\nu}{\mu}\right)$ in (2.2), then

$$
\mu \leqq \rho \tau \exp \left(1-\frac{\nu}{\mu}\right)
$$

Again, as $e^{x} \geqq e x$ for all $x$ we have

$$
\mu e \frac{\nu}{\mu} \leqq \mu e^{\nu / \mu} \leqq e \rho \tau
$$

Or

$$
\nu \leqq \frac{\mu}{e} e^{\nu / \mu} \leqq \rho \pi
$$

Taking $k=1$ in (2.4) we get $\rho \tau \leqq \mu$. From the right hand inequality of (2.7) we get

$$
e \rho \tau \geqq \mu e^{\nu / \mu} \geqq \mu\left\{1+\frac{\nu}{\mu}\right\}
$$

and finally we obtain

and

$$
\nu \leqq \frac{\mu}{e} e^{\nu / \mu} \leqq \rho \tau \leqq \mu \leqq e \rho \tau
$$

$$
\nu+\mu \leqq e \rho \tau .
$$

Proof of CoRollary. If $\nu=\rho$ from (2.7) we have $\mu e^{\nu / \mu} \leqq e \nu$.

Or

$$
e^{\nu / \mu} \leqq e \frac{\nu}{\mu}
$$

and since $e^{x} \geqq e x$ for all $x$ and the equality holds only if $x=1 \quad e^{\nu / \mu}<e \frac{\nu}{\mu}$ is not possible. Hence $e^{\nu / \mu}=e \frac{\nu}{\mu}$, i. e., $\nu=\mu$. If $\mu=\nu$ again from (2.7) we get

While

$$
e \mu=e \nu \leqq e \rho \tau,
$$

Hence

$$
\begin{gathered}
\mu \geqq \rho \tau . \\
\mu=\nu=\rho \tau .
\end{gathered}
$$

Next, if $\nu=\rho \tau$ which implies $\mu=\rho \tau$. So $\mu+\nu=2 \rho \tau<e \rho \tau$. Now, let 
$\mu+\nu=e \rho \tau$ then $\nu$ will be less than $\rho \tau$ for if it were equal to $\rho \tau$ then $\mu+\nu$ will have to be less than $e \rho \tau$. $\therefore$ Contradiction. Hence the result.

3. We remark that:

(i) If $\mu=0$ then $\tau=0$ and conversely.

(ii) If $\nu=\infty$ then $t=\infty$.

(iii) If $t=\infty$ then $\mu=\infty$.

(iv) If $\nu=0, \mu<\infty$ then $t=0$.

(v) If $t=0$ then $\nu=0$.

\section{REFERENCES}

[1] G. H. HARDY AND W. W. Rogosinski, Notes on Fourier Series (III), Quart. Journ. of Math. (Oxford Series) 16(1945), 49-58.

[2] R. NEvantinNa, Eindeutige Analytische Funktionen, 2 Aufl. (195̄3), Springer Verlag, Berlin.

[3] S.K. SingH, Thesis on Entire and Meromorphic Functions (1953). The Aligarh Muslim University, Aligarh.

Hindu College Moradabad, India. 